\title{
ON THE PROGENITOR OF THE TYPE II-PLATEAU SN 2008cn in NGC 4603*
}

\author{
Nancy Elias-Rosa ${ }^{1}$, Schuyler D. Van Dyk ${ }^{1}$, Weidong Li $^{2}$, Nidia Morrell ${ }^{3}$, Sergio Gonzalez ${ }^{3}$, Mario Hamuy ${ }^{4}$, \\ Alexei V. Filippenko ${ }^{2}$, Jean-Charles Cuillandre 5 , Ryan J. Foley ${ }^{2,6,7}$, and Nathan Smith ${ }^{2}$ \\ ${ }^{1}$ Spitzer Science Center, California Institute of Technology, 1200 E. California Blvd., Pasadena, CA 91125, USA; nelias@ ipac.caltech.edu, \\ vandyk@ipac.caltech.edu. \\ ${ }^{2}$ Department of Astronomy, University of California, Berkeley, CA 94720-3411, USA \\ ${ }^{3}$ Las Campanas Observatory, Carnegie Observatories, Casilla 601, La Serena, Chile \\ ${ }^{4}$ Departamento de Astronomia, Universidad de Chile, Casilla 36-D, Santiago, Chile \\ ${ }^{5}$ Canada France-Hawaii Telescope Corporation, 65-1238 Mamalahoa Hwy, Kamuela, HI 96743, USA \\ ${ }^{6}$ Harvard/Smithsonian Center for Astrophysics, 60 Garden Street, Cambridge, MA 02138, USA \\ Received 2009 March 24; accepted 2009 October 14; published 2009 November 10
}

\begin{abstract}
A trend is emerging regarding the progenitor stars that give rise to the most common core-collapse supernovae (SNe), those of Type II-Plateau (II-P): they generally appear to be red supergiants with a limited range of initial masses, $\sim 8-16 M_{\odot}$. Here, we consider another example, SN $2008 \mathrm{cn}$, in the nearly face-on spiral galaxy NGC 4603. Even with limited photometric data, it appears that SN 2008cn is not a normal SN II-P, but is of the high-luminosity subclass. Through comparison of pre- and post-explosion images obtained with the Wide Field and Planetary Camera 2 on board the Hubble Space Telescope, we have isolated a supergiant star prior to explosion at nearly the same position as the SN. We provide evidence that this supergiant may well be the progenitor of the SN, although this identification is not entirely unambiguous. This is exacerbated by the distance to the host galaxy, 33.3 Mpc, making SN 2008cn the most distant SN II-P yet for which an attempt has been made to identify a progenitor star in pre-SN images. The progenitor candidate has a more yellow color ( $[V-I]_{0}=0.98$ mag and $T_{\text {eff }}=5200 \pm 300 \mathrm{~K}$ ) than generally would be expected and, if a single star, would require that it exploded during a "blue loop" evolutionary phase, which is theoretically not expected to occur. Nonetheless, we estimate an initial mass of $M_{\mathrm{ini}}=15 \pm 2 M_{\odot}$ for this star, which is within the expected mass range for SN II-P progenitors. The yellower color could also arise from the blend of two or more stars, such as a red supergiant and a brighter, blue supergiant. Such a red supergiant hidden in this blend could instead be the progenitor and would also have an initial mass within the expected progenitor mass range. Furthermore, the yellow supergiant could be in a massive, interacting binary system, analogous to the possible yellow supergiant progenitor of the high-luminosity SN II-P 2004et. Finally, if the yellow supergiant is not the progenitor, or is not a stellar blend or binary containing the progenitor, then we constrain any undetected progenitor star to be a red supergiant with $M_{\text {ini }} \lesssim 11 M_{\odot}$, considering a physically more realistic scenario of explosion at the model endpoint luminosity for a rotating star.
\end{abstract}

Key words: galaxies: individual (NGC 4603) - stars: evolution - supernovae: general - supernovae: individual (SN 2008cn)

\section{INTRODUCTION}

Massive stars are thought to evolve to an end state which results in the collapse of the stellar core, as the hydrostatic pressure in the core can no longer support gravity. Although the physics of what follows is still uncertain, it appears that, following core collapse, the pressure due to neutrinos released from the core results in the explosion of the rest of the stara supernova (SN; e.g., Marek \& Janka 2009). Core-collapse supernovae (CC-SNe) have been at the forefront of astronomical research for the better part of a century, and yet we still do not have complete knowledge of which stars explode. It is possible that the relative heterogeneity in observed properties of CC-SNe arises from a range of massive progenitors. Although indirect evidence (spectral features, light-curve shapes, environmental information) provides some clues, direct identification of the star before the SN explosion (e.g., Van Dyk et al. 1999, 2000;

\footnotetext{
* Based on observations made with the NASA/ESA Hubble Space Telescope, obtained from the Data Archive at the Space Telescope Science Institute, which is operated by the Association of Universities for Research in Astronomy, Inc., under NASA contract NAS 05-26555. Based in part on data gathered with the $6.5 \mathrm{~m}$ Magellan telescopes, located at Las Campanas Observatory, Chile.

7 Clay Fellow
}

Smartt 2002) is the more precise means of determining the nature of the progenitor star.

At this time of writing, the only kind of $\mathrm{CC}-\mathrm{SNe}$ for which progenitors have been directly identified is the Type II-Plateau supernovae (SNe II-P; e.g., Van Dyk et al. 2003; Li et al. 2006), with the exception of SN $2005 \mathrm{gl}$ in NGC 266, a Type II-narrow supernova (SN IIn) for which a progenitor may well have been identified (Gal-Yam et al. 2007; Gal-Yam \& Leonard 2009). The SNe II-P exhibit Balmer lines with prominent P Cygnilike profiles in their optical spectra at early times. Their optical light curve shape shows a characteristic "plateau" phase, prior to an exponential tail (e.g., Barbon et al. 1979). The trend is for their progenitor masses to be in the range of 8-20 $M_{\odot}$ ( $\mathrm{Li}$ et al. 2006). Based on the statistics of $20 \mathrm{SNe}$ II-P for which progenitors have been isolated or upper mass limits established, Smartt et al. (2009) derive a more limited range of $8.5\left(_{-1.5}^{+1}\right) \lesssim M_{\text {ZAMS }}\left(M_{\odot}\right) \lesssim 16.5( \pm 1.5)$ for these stars. All of these progenitors exploded in the red supergiant (RSG) phase, as we would theoretically expect, except possibly for SN 2004et (Li et al. 2005) and SN 2006ov (Li et al. 2007). Given that fewer than 10 progenitors for SNe II-P have actually been directly identified, the addition of further examples is essential for validating this trend and further constraining stellar evolutionary theory. 
Table 1

Imaging Data for the SN 2008cn Site

\begin{tabular}{|c|c|c|c|c|c|}
\hline UT Date & Telescope/Instrument & Filter & $\begin{array}{l}\text { Exp. Time } \\
\text { (s) }\end{array}$ & $\begin{array}{l}\text { No. of Images or } \\
\text { CR-SPLIT Pairs }\end{array}$ & $\begin{array}{l}H S T \text { Image } \\
\text { Sets }\end{array}$ \\
\hline \multicolumn{6}{|c|}{ Pre-explosion } \\
\hline 1996 May 27 & $H S T / W P F C 2$ & F555W & $900 / 1300$ & 3 & u381010[1-6]t \\
\hline 1996 Jun 1 & $H S T / \mathrm{WPFC} 2$ & F555W & $900 / 1300$ & 3 & u381020[1-6]t \\
\hline 1996 Jun 8 & $H S T / \mathrm{WPFC} 2$ & F555W & $900 / 1300$ & 3 & u381030[1-6]t \\
\hline 1996 Jun 14 & $H S T / \mathrm{WPFC} 2$ & F555W & $900 / 1300$ & 3 & u381040[1-6]t \\
\hline 1996 Jun 20 & $H S T / \mathrm{WPFC} 2$ & F555W & $900 / 1300$ & 3 & $\mathrm{u} 381050[1-6] \mathrm{t}$ \\
\hline 1996 Jun 28 & $H S T / \mathrm{WPFC} 2$ & F555W & $900 / 1300$ & 3 & u381060[1-6]f \\
\hline $1996 \mathrm{Jul} 7$ & $H S T / \mathrm{WPFC} 2$ & F555W & $900 / 1300$ & 2 & u381070[1-4]f \\
\hline 1997 Jun 17 & $H S T / \mathrm{WPFC} 2$ & F555W & $1100 / 1300$ & 2 & u381080[1-4]f \\
\hline 1997 Jun 17 & $H S T / \mathrm{WPFC} 2$ & F814W & $1100 / 1300$ & 3 & $\mathrm{u} 3 \mathrm{zc} 010[1-6] \mathrm{m}$ \\
\hline 1997 Jul 17 & $H S T / \mathrm{WPFC} 2$ & F814W & $1100 / 1300$ & 3 & $\mathrm{u} 3 \mathrm{zc} 020[1-6] \mathrm{m}$ \\
\hline 1997 Jul 18 & HST/WPFC2 & F555W & $1100 / 1300$ & 2 & u381090[1-4]f \\
\hline \multicolumn{6}{|c|}{ Post-explosion } \\
\hline 2008 Jun 1 & Swope/CCD & $V$ & 100 & 1 & \\
\hline 2008 Jun 15 & MagI/IMACS & $V$ & 100 & 2 & $\ldots$ \\
\hline 2008 Aug 26 & $H S T / \mathrm{WPFC} 2^{\mathrm{a}}$ & F555W & $1100 / 1300$ & 7 & ua22030[1-7]m \\
\hline 2008 Aug 26 & $H S T / \mathrm{WPFC} 2^{\mathrm{a}}$ & F814W & $1100 / 1300$ & 8 & ua220308-9/a-fm \\
\hline 2009 Jul 1 & CTIO/ANDICAM & $V R I$ & 360 & $3 / 2 / 2$ & $\ldots$ \\
\hline
\end{tabular}

Notes. HST/WPFC2: Hubble Space Telescope + Wide Field Planetary Camera 2, WF2 chip, 0" 1 pixel ${ }^{-1}$; Swope/CCD: $1.0 \mathrm{~m}$ Swope telescope + Direct CCD Camera, 0". 435 pixel $^{-1}$; MagI/IMACS: Baade $6.5 \mathrm{~m}$ Magellan Telescope + IMACS Long-Camera, $0^{\prime \prime} 2$ pixel $^{-1}$; CTIO/ANDICAM: CTIO $1.3 \mathrm{~m}$ Telescope + ANDICAM Camera, 0!'369 pixel $^{-1}$.

a In these observations, SN $2008 \mathrm{cn}$ is located on the PC chip, $0^{\prime \prime} 046 \mathrm{pixel}^{-1}$.

The explosion of SN 2008cn provides us with an opportunity to increase the sample, including particularly the highluminosity SN subclass (e.g., Pastorello 2003), and to extend our techniques to the study of stars which have exploded in relatively more distant galaxies.

Martin \& Monard (2008) discovered SN 2008cn on 2008 May 21.52 (UT dates are used throughout this paper), at 4."7E and $23 . \prime 2 \mathrm{~N}$ of the center of the nearby $(c z=2592$ $\mathrm{km} \mathrm{s}^{-1}$ ), nearly face-on spiral galaxy NGC 4603. Nine days later, Stritzinger \& Morrell (2008) classified the SN as Type II, a few days past explosion. Li et al. (2008) measured a precise position for a putative progenitor of SN 2008cn as $\alpha=$ $12^{\mathrm{h}} 40^{\mathrm{m}} 55^{\mathrm{s}} .64, \delta=-40^{\circ} 58^{\prime} 12^{\prime \prime} \cdot 9$ (J2000.0), from high-quality Hubble Space Telescope (HST) archival images of the host galaxy taken with the Wide Field Planetary Camera 2 (WFPC2). The position was established after the HST images were registered with co-added $V$-band images taken with the $1.0 \mathrm{~m}$ Swope telescope at Las Campanas Observatory on 2008 June 1.15 (a geometrical transformation was performed, resulting in an accuracy of $0{ }^{\prime} 1$ on the WFPC 2 images). The claim was that the detected object could be a RSG with an absolute $I$-band magnitude of -7.9 and an intrinsic $V-I$ color of $1.2 \mathrm{mag}$.

Here, we report on a more detailed analysis of this object. In Section 2, we determine the characteristics of SN $2008 \mathrm{cn}$. Section 3 explains the process of identification and analysis of the possible progenitor using HST images taken both before, and after, the SN explosion. We discuss the nature of the progenitor, based on this analysis, in Section 4, and in Section 5 we summarize our conclusions.

\section{THE NATURE OF SN $2008 \mathrm{CN}$}

Before discussing the nature of the progenitor of SN 2008cn, we should derive more information from observations of the SN itself (its spectrum and light curves). Unfortunately, we have only scant coverage, but even from this partial data set we are able to produce a relatively consistent reddening estimate and can characterize SN $2008 \mathrm{cn}$.

In addition to the images obtained with the Swope $1.0 \mathrm{~m}$ telescope on 2008 June 1, $V$-band images of the SN were also obtained on 2008 June 15 with the Baade $6.5 \mathrm{~m}$ Magellan telescope + the Inamori-Magellan Areal Camera \& Spectrograph (IMACS) at Las Campanas Observatory; see Table 1. The images were trimmed, corrected for overscan and bias, and flat-fielded. Instrumental magnitudes for the SN and the stars in its immediate environment were measured using SNOoPY (SuperNOvaPhotometrY), ${ }^{8}$ which is based on DAOPHOT (Stetson 1987) within IRAF ${ }^{9}$ and allows for fitting of the stellar profiles with a point-spread function (PSF) created from a set of unsaturated stars in the images. The SN magnitudes were calibrated using the comparison stars in the images, based on four other photometric nights when the SN was observed (C. Contreras 2008, private communication). We also obtained latetime photometry for the SN from our HST Target of Opportunity (ToO) observations (see Section 3) and a late-time upper limit ( $\sim 416$ days after explosion) from images obtained on 2009 July 01.9 with the Cerro Tololo Inter-American Observatory (CTIO) $1.3 \mathrm{~m}$ telescope + ANDICAM, operated by the SMARTS consortium. The SN photometry is listed in Table 3.

We have also analyzed an unpublished high-resolution optical spectrum of the SN, obtained on 2008 June 16 with the Baade $6.5 \mathrm{~m}$ Magellan telescope + IMACS. The complete set of ground-based observations, including this spectrum, will be presented elsewhere by the Carnegie Supernova Project

\footnotetext{
8 SNOoPY is a package originally designed by F. Patat and later implemented in IRAF by E. Cappellaro.

9 IRAF (Image Reduction and Analysis Facility) is distributed by the NOAO, which is operated by the Association of Universities for Research in Astronomy, Inc., under cooperative agreement with the National Science Foundation.
} 
[CSP.${ }^{10}$ The spectrum appears quite similar to that of the highluminosity SN 1996W (Pastorello 2003, C. Inserra et al. 2009, in preparation) at $\sim$ one month after explosion. The SN $2008 \mathrm{cn}$ spectrum exhibits similar P Cygni profiles for the H Balmer emission lines; however, it lacks He I $\lambda 5876$, which is seen in the SN 1996W spectrum at this phase. We derive an estimate of the explosion date (JD $2454598 \pm 2$ ), which is consistent with the date of the upper limit to the $\mathrm{SN}$ detection (May 8.8) given by Martin \& Monard (2008). SN 2008cn also presents similarities with the spectra of other high-luminosity events, such as $\mathrm{SNe}$ 1992H (Clocchiatti et al. 1996) and 2003hn (Krisciunas et al. 2009) at coeval epochs, even if these last two show a more evolved Ca II near-infrared triplet (Ca II $\lambda \lambda 8498,8542,8662$ ). In general, as has been discussed by Pastorello (2003), all of these high-luminosity SN spectra show a slower evolution, compared with those of intermediate luminosity, such as SN 1999em (Hamuy et al. 2001; Leonard et al. 2002; Elmhamdi et al. 2003).

Next, we estimated the reddening to SN $2008 \mathrm{cn}$ from the spectrum. Measuring the equivalent width $(\mathrm{EW})$ of the Na I D line at the host-galaxy redshift $(z=0.009)$ in the spectrum, we find that $\mathrm{EW}(\mathrm{Na}$ I D) $=1.7 \AA$. Using the relation between extinction and EW(Na I D) from N. Elias-Rosa et al. (2009, in preparation), and assuming the Cardelli et al. (1989) reddening law with updated wavelengths (as discussed by N. Elias-Rosa et al. 2009, in preparation) and a Galactic foreground $E(V-I)=$ $0.22 \mathrm{mag}$ (Schlegel et al. 1998), we derive $E(V-I)_{\mathrm{tot}}=$ $0.44 \pm 0.06 \mathrm{mag}\left(E[B-V]_{\mathrm{tot}}=0.35 \pm 0.04 \mathrm{mag}\right)$. We adopt this value for $\mathrm{SN} 2008 \mathrm{cn}$.

Adopting the distance to NGC 4603 determined by Newman et al. (1999) using Cepheid variables, $33.3 \pm 0.2 \mathrm{Mpc}$, together with our extinction estimate and the explosion date suggested by the spectrum, we have compared our $V$ absolute light curve, however limited, with those of $\mathrm{SNe} 1992 \mathrm{H}$ (Clocchiatti et al. 1996), 1996W (Pastorello 2003, C. Inserra et al. 2009, in preparation), 1999em (Hamuy et al. 2001; Leonard et al. 2002; Elmhamdi et al. 2003), 2003hn (Krisciunas et al. 2009), and 2004et (Sahu et al. 2006); see Figure 1 (top). All of these latter $\mathrm{SNe}$ are likely of high luminosity, except for SN $1999 \mathrm{em}$. The overall shape of the light curves of SNe 2008cn and 2003hn are very similar, and the length of the plateau for each is also almost the same. SN $2008 \mathrm{cn}$ is less luminous than the other highluminosity SNe II-P, but it is clearly more luminous (by $\sim 0.5$ mag) than SN 1999em. The mean absolute $V$ magnitude during the plateau phase, estimated by averaging the magnitudes from 20 to 35 days after explosion, is -17.23 , brighter than $\sim 90 \%$ of the SNe II-P analyzed in a complete sample by W. Li et al. (2009, in preparation). From our data, the SN 2008cn plateau may well be as steep as that of SN 2003hn. Unfortunately, we were not able to compare properly the SN $2008 \mathrm{cn}$ light curve with that of SN 1996W, given the lack of observations for this latter $\mathrm{SN}$ at the end of the plateau phase.

In Figure 1 (bottom), we can also see the comparison of the intrinsic $(V-I)_{0}$ color for SN $2008 \mathrm{cn}$, from our late-time $H S T$ observations (see Section 3), to $(V-I)_{0}$ for the other $\mathrm{SNe}$ considered above. These latter SN colors have been corrected for extinction using published estimates and assuming the Cardelli et al. (1989) extinction law. In the case of SN 2008cn, we have corrected the observed color by the reddening discussed above.

Both the spectroscopy and the photometry of SN $2008 \mathrm{cn}$ indicate that it is a highly luminous SN II-P. For only one

\footnotetext{
$10 \mathrm{http}: / / \mathrm{csp} 1.1 \mathrm{co} . \mathrm{cl} / \mathrm{cspuser1/PUB/CSP.html}$
}
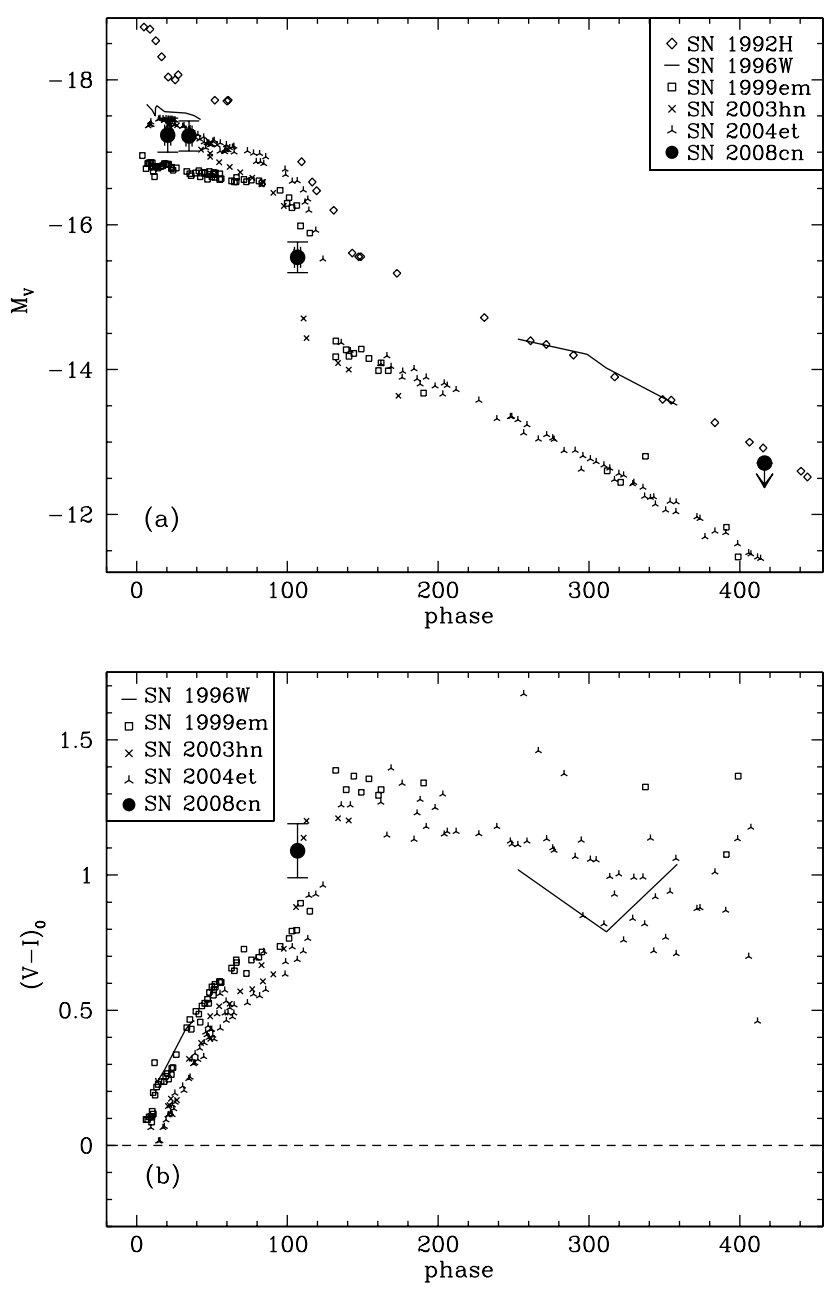

Figure 1. Top: absolute $V$ light curve of SN $2008 \mathrm{cn}$ (filled circle) along with those of the high-luminosity SNe II-P 1992H (diamonds), 1996W (solid line), 2003hn (crosses), and 2004et (pentagons), as well as of the normal SN IIP 1999em (squares). Distances and extinction estimates for these latter $\mathrm{SNe}$ have been adopted from the literature. Bottom: the intrinsic $(V-I)_{0}$ color of SN $2008 \mathrm{cn}$, compared with the intrinsic color evolution of the latter SNe. The color of SN 2008cn has been corrected for the assumed reddening, $E(V-I)=$ $0.44 \mathrm{mag}$. See the text for additional details.

other SN of this kind, SN 2004et, has a progenitor star been identified (Li et al. 2005; but see Smartt et al. 2009). As discussed by Pastorello (2003), semianalytical models predict that these $\mathrm{SNe}$ should have massive progenitors, near the high end of the mass range for SNe II-P determined by Smartt et al. (2009). Thus, locating the progenitor of SN $2008 \mathrm{cn}$ could enrich our knowledge of the progenitor stars for these unusual SNe.

\section{IDENTIFICATION AND ANALYSIS OF THE PROGENITOR CANDIDATE}

As can be seen in the next subsections, the analysis of the location of the possible progenitor of SN 2008cn was developed following several steps: (1) search the HST archive for pre-SN images of the $\mathrm{SN}$; (2) locate the progenitor candidate in these pre-explosion images, assisted by ground-based images of the SN at early times; and (3) confirm the candidate, first identified by Li et al. (2008), using post-explosion HST images obtained as part of our ToO program GO-11119.

\subsection{Isolating the Progenitor Candidate}

Once SN 2008cn was discovered, we found 48 images in the F555W $(\sim V)$ and 12 images in F814W $(\sim I)$ filters in the HST 


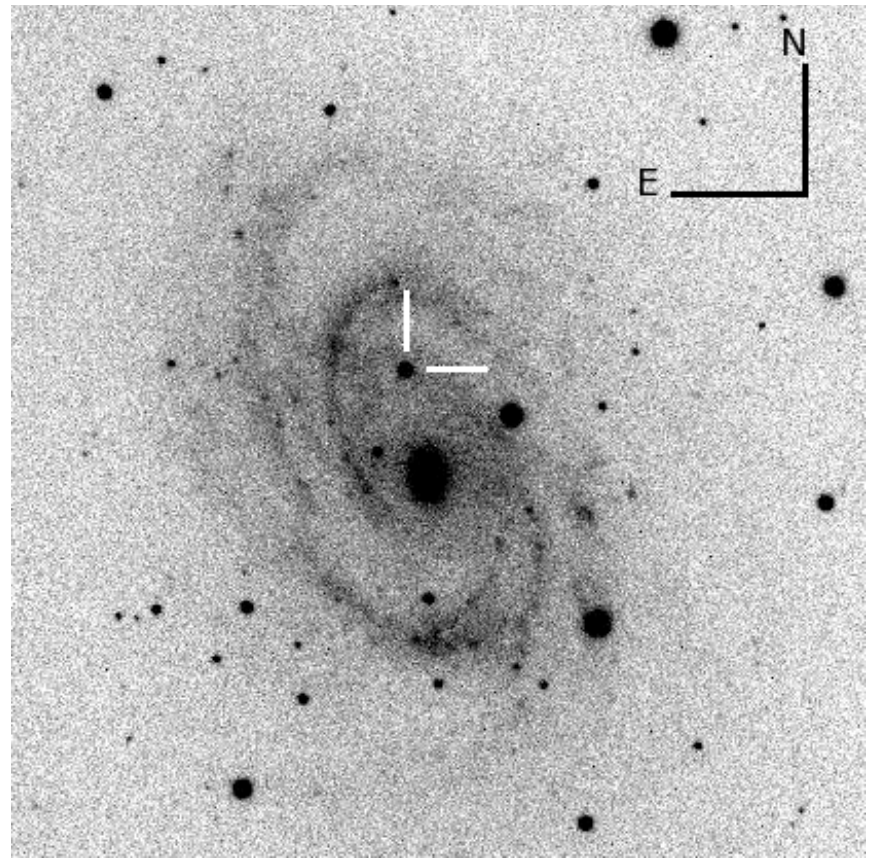

Figure 2. Baade $6.5 \mathrm{~m}$ Magellan Telescope + IMACS $V$-band image of SN $2008 \mathrm{cn}$. The SN is indicated by the white arrows. The field of view is $\sim 4^{\prime} \times 4^{\prime}$.

archive. ${ }^{11}$ These images were all obtained with WFPC2 between 1996 and 1997 by program GO-6439 (PI: S. Zepf) in order to determine the distance to NGC 4603 using Cepheid variables (Newman et al. 1999). At each epoch, images in each band were obtained in cosmic-ray split (CR-SPLIT) pairs; see Table 1 for a complete list of the pre-explosion observations analyzed here. In all of these images the site of SN $2008 \mathrm{cn}$ was located on the WF2 chip (pixel scale of $0{ }^{\prime \prime} 1$ pixel $^{-1}$ ). The exposure times in each band were in the range of 900-1300 s. Combining the images in each band over all epochs of the Cepheid observations yields a total image depth of $\sim 16 \mathrm{hr}$ in F555W and $\sim 4 \mathrm{hr}$ in F814W.

To obtain a more accurate position of the SN 2008cn progenitor candidate in the archival HST images than what Li et al. (2008) were able to accomplish with the $1.0 \mathrm{~m}$ telescope images, we compared the HST images with the Magellan V-band image of the SN (with pixel scale 0.28 pixel $^{-1}$ ) from 2008 June 15; see Figure 2. We combined two of the CR-SPLIT HST exposures in F555W (images u3810101t, u3810102t) in order to reject cosmicray hits, and produced a $1600 \times 1600$ pixel mosaic of all four chips from the combined image, using the routines crrej and wmosaic of the STSDAS package within IRAF. We then used the task rotate within IRAF to rotate the mosaic around its center (pixel 800,800 ) by the angle defined in the mosaic header keyword ORIENTAT. We identified 7-11 point-like sources in common between the mosaic and the Magellan image and measured their pixel coordinates with imexamine. Using the IRAF task geomap, we carried out a geometrical transformation between the two sets of coordinates, with root mean square (rms) uncertainty $\lesssim 0^{\prime \prime}$. 1 . With the IRAF task geotran we registered the Magellan $V$ image to the coordinate frame of the rotated $H S T$ F555W mosaic, which allowed us to measure a precise pixel position for the $\mathrm{SN}$ in this latter image at [1132.58, 843.99]. Registering available point sources to the reference frame of the Two

\footnotetext{
11 http://archive.stsci.edu/hst/
}

Micron All Sky Survey (2MASS ${ }^{12}$ ) Point Source Catalog, this pixel position corresponds to $\alpha=12^{\mathrm{h}} 40^{\mathrm{m}} 55^{\mathrm{s}} .64, \delta=$ $-40^{\circ} 58^{\prime} 12^{\prime \prime}$. 1 (J2000.0). Considering the 2MASS positional rms uncertainty of $\sim 0^{\prime} .2$, the total uncertainties estimated in this position are $\Delta \alpha=0,2$ and $\Delta \delta=0.3$.

Through careful examination of the image mosaic, the object nearest the $\mathrm{SN}$ site, as determined from images obtained in both $H S T$ filters, is located at $\alpha=12^{\mathrm{h}} 40^{\mathrm{m}} 55^{\mathrm{s}} .63, \delta=-40^{\circ} 58^{\prime} 12^{\prime \prime} .2$ (pixel [1134.37, 843.00]). We consider this object, the same as that suggested by Li et al. (2008), to be the progenitor candidate. Given that the offset between the SN position (measured from the ground-based image) and the candidate position is significant $(\Delta x=1.79, \Delta y=0.99)$, it became essential that we use even higher-resolution images of the SN (ideally, at the same resolution as the pre-SN HST image) to attempt to reduce this offset, before further considering this candidate as the actual progenitor star.

\subsection{Confirmation of the Progenitor Location}

Post-explosion ToO HST WFPC2 observations were obtained in F555W and F814W on 2008 August 26 (see Table 1 for more details) as part of our program GO-11119. The SN was imaged on the PC chip (pixel scale $0^{\prime \prime} .045$ pixel $^{-1}$ ). The SN was still quite bright at the time of these observations, so we used the gain setting of $14 \mathrm{e}^{-} \mathrm{DN}^{-1}$. Furthermore, we obtained a set of images in each band with short exposure times, with no dithering between images, and each set was combined into a single image per band (see below).

To achieve high-precision relative astrometry between the $\mathrm{SN}$ and progenitor candidate positions, we needed to geometrically transform these post-explosion images to match the preexplosion ones. Pre-explosion image mosaics for each band, which have been combined from the individual exposures (see Section 3.1) to the pixel scale of the PC chip using the drizzle algorithm (Fruchter \& Hook 2002), are available from the HST Legacy Archive. ${ }^{13}$ The drizzle image processing method weights input images according to the statistical significance of each pixel, and removes the effects of geometric distortion on both image shape and photometry. In addition, it combines dithered images in the presence of cosmic rays and improves the resolution of the mosaic. Similarly, we "drizzled" the post-explosion images to the PC chip resolution, following the recipe provided by the Space Telescope Science Institute. ${ }^{14}$ The task drizzle runs under PYRAF as part of the STSDAS package. Next, the registration of the pre-explosion and post-explosion image mosaics was performed using eleven point-like sources in common between the two sets of data. The number of available fiducial stars is limited by the relatively shallow depth of the combined ToO exposures. The fiducial sources are faint; however, they are concentrated around the SN site, reducing the impact of any possible residual distortion remaining in the larger mosaics. Comparing pixel-by-pixel both sets of data, we verified the proximity of the point-like progenitor candidate in the pre-explosion images very near the location of the SN; see Figure 3. Using two methods, the task daofind within IRAF/DAOPHOT and imexamine, we measured the positions of both the $\mathrm{SN}$ and progenitor candidate. We averaged the results from both methods to establish final pixel positions, which are [2458.13, 2045.74] and [2457.86, 2045.22]

\footnotetext{
12 http://www.ipac.caltech.edu/2mass/

13 http://archive.stsci.edu/hlsp/

14 http://www.stsci.edu/hst/wfpc2/analysis/drizzle.html
} 

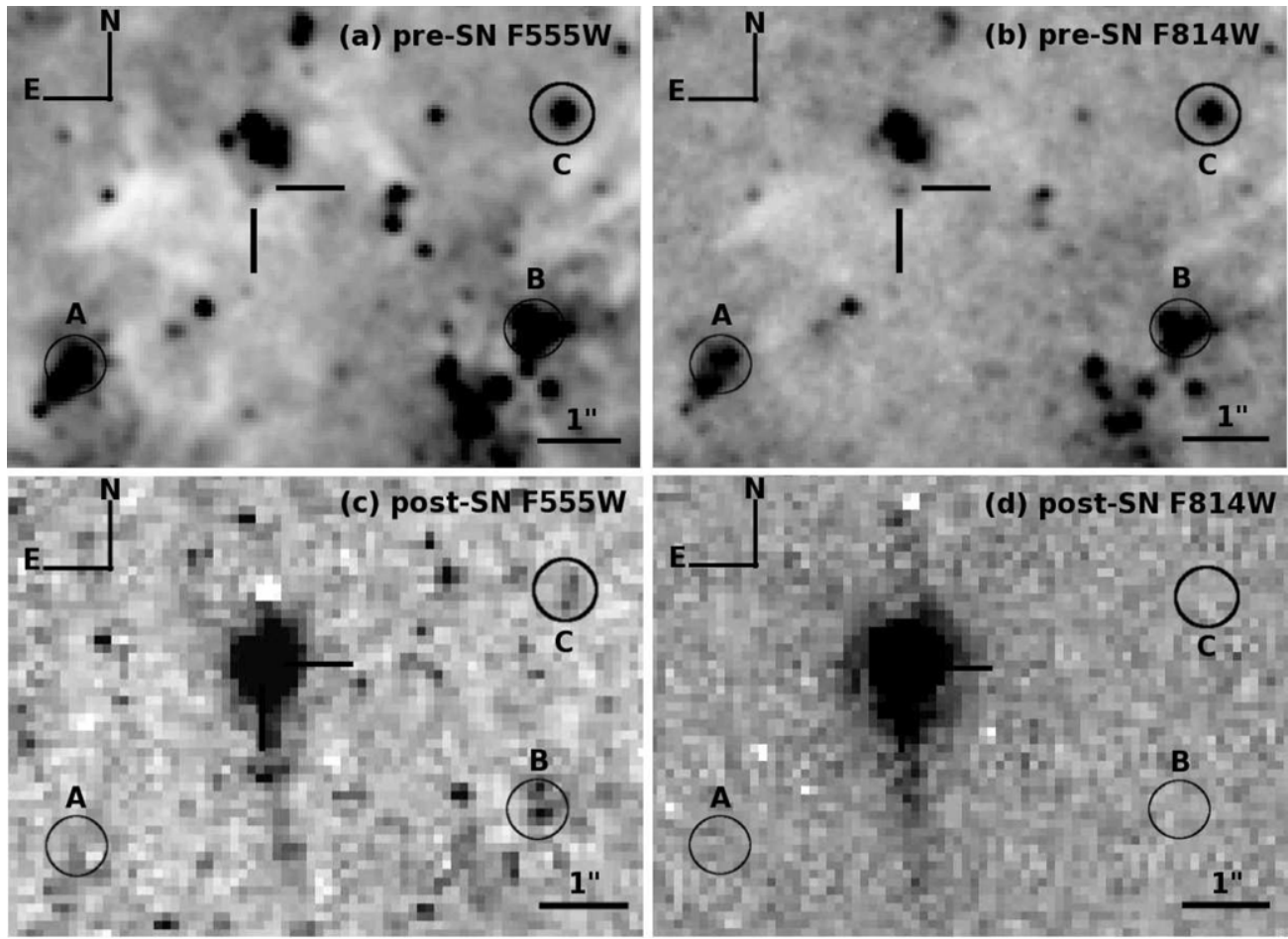

Figure 3. Subsections of the HST + WFPC2 images, in F555W (left) and F814W (right), of NGC 4603 before (panels (a) and (b)) and after (panels (c) and (d)) the SN 2008cn explosion. The positions of the SN candidate progenitor and SN are indicated by bars. The three objects "A," "B," and "C" were not used in the image astrometry, due to their relative faintness; they are labeled only to aid the eye in locating the progenitor in the field.

Table 2

Differences Between the SN and Progenitor Candidate Positions

\begin{tabular}{lcc}
\hline \hline \multicolumn{1}{c}{ Angular Quantity } & $V(\alpha / \delta)$ & $I(\alpha / \delta)$ \\
\hline Uncertainty in the progenitor position (mas) & $7 / 3$ & $0 / 2$ \\
Uncertainty in the SN position (mas) & $2 / 1$ & $4 / 0$ \\
Geometric transformation (mas) & $29 / 28$ & $29 / 21$ \\
Total uncertainty (mas) & $30 / 28$ & $29 / 21$ \\
Difference in position (mas) & $9 / 40$ & $5 / 33$ \\
\hline
\end{tabular}

Notes. Uncertainties in the $\mathrm{SN}$ and candidate right ascension, $\alpha$, and declination, $\delta$, in milliarcsec, were estimated as the standard deviation of the average. Geometric transformation errors are derived from the positional differences between the fiducial stars used in the transformation. The total uncertainty is the quadrature sum of these uncertainties. The last line lists the residual difference between the $\mathrm{SN}$ and progenitor position after the geometric transformation.

for the SN and [2457.94, 2046.54] and [2457.96, 2045.89] for the progenitor candidate, in F555W and F814W, respectively. Note that no other source was located within a $1.5 \sigma$ ( $\leqslant 2$ pixels) radius from the progenitor candidate position. The differences between the $\mathrm{SN}$ and the progenitor candidate positions, compared with the total estimated uncertainty in the astrometry, are given in Table 2. This latter uncertainty was calculated as a quadrature sum of the uncertainties in the SN and progenitor candidate positions, and the rms uncertainty in the geometric transformation.

From the results in Table 2 it can be seen that the difference between the $\mathrm{SN}$ position and the position of the progenitor candidate in declination is somewhat larger than the total astrometric error, for both bands. Although the relative positional coincidence between the candidate and SN position in right ascension suggests that we have indeed identified the SN progenitor star, the small offset in declination somewhat weakens our confidence. We considered it possible that the presence of the two, much brighter point sources within $\sim 1^{\prime \prime}$ to the north of the candidate (Figure 3, panels (a) and (b)) may be affecting the measurement of the centroid in declination, resulting in a northward skew in the progenitor candidate position using both of our methods. For this reason, we repeated the measurements described above after first masking out these two bright stars in the pre-explosion images. Doing so, however, did not significantly reduce the positional difference in declination between the candidate and the $\mathrm{SN}$ in either band.

For now, we make the assumption that the candidate is the progenitor star of SN 2008cn and discuss below the implications of this assumption. However, given the slight positional difference, we will also subsequently consider the implications if this star is not the $\mathrm{SN}$ progenitor.

\subsection{Photometry of the Pre- and Post-explosion HST Images}

We obtained photometry of both the progenitor candidate in the pre-explosion $H S T$ images and the $\mathrm{SN}$ in the post-explosion $H S T$ images. Photometry of all of the WFPC2 images was performed using the package HSTphot ${ }^{15}$ (Dolphin 2000a). Since our pre-explosion images were obtained at different epochs and at various pointings, and not always in matched pairs of bands, we measured the relative offsets between the pre-explosion images (the orientation angle for all of these HST images is 143.89). Once corrected for relative offsets with respect to one fiducial image, we ran the package on the CR-SPLIT pairs of the individual frames in each band separately, with option flag 10, which combines turning on the local sky determination, turning off empirically determined aperture corrections (using default values instead), and turning on PSF residual determination, with a total detection threshold of $3 \sigma$. We then transformed

\footnotetext{
15 HSTphot is a stellar photometry package specifically designed for use with HST WFPC2 images. We used version $1.1 .7 \mathrm{~b}$, updated 2008 July 19
} 
Table 3

Photometry of SN 2008cn, the Progenitor Candidate, and Artificial Stars

\begin{tabular}{|c|c|c|c|c|c|}
\hline Object & UT Date & $\begin{array}{c}\text { F555W } \\
\text { (mag) }\end{array}$ & $\begin{array}{c}\text { F814W } \\
\text { (mag) }\end{array}$ & $\begin{array}{c}V \\
(\mathrm{mag})\end{array}$ & $\begin{array}{c}I \\
(\mathrm{mag})\end{array}$ \\
\hline SN 2008cn & 2008 Jun 1 & $\ldots$ & $\ldots$ & $16.51 \pm 0.22$ & $\ldots$ \\
\hline SN 2008cn & 2008 Jun 15 & $\ldots$ & $\ldots$ & $16.46 \pm 0.02$ & $\ldots$ \\
\hline SN $2008 \mathrm{cn}$ & 2008 Aug 26 & $18.15 \pm 0.01$ & $16.64 \pm 0.01$ & $18.13 \pm 0.04$ & $16.60 \pm 0.05$ \\
\hline SN $2008 \mathrm{cn}$ & 2009 Jul 1 & $\ldots$ & $\ldots$ & $\gtrsim 21.0$ & $\gtrsim 20.5$ \\
\hline Progenitor candidate & $\ldots$ & $26.41 \pm 0.04$ & $25.01 \pm 0.06$ & $26.37 \pm 0.04$ & $24.96 \pm 0.07$ \\
\hline Artificial star limit ${ }^{\mathrm{a}}$ & $\cdots$ & $\gtrsim 28.1$ & $\gtrsim 25.4$ & $\gtrsim 28.0$ & $\gtrsim 25.4$ \\
\hline
\end{tabular}

Note. ${ }^{\text {a }}$ The $2 \sigma$ upper limits to the magnitudes for the undetected artificial stars are the averages of the determinations from each set of CR-SPLIT pairs. Uncertainties were estimated in the photometric calibration for the ground-based observations, and by HSTphot for the HST images.

the flight-system magnitudes in $\mathrm{F} 555 \mathrm{~W}$ and $\mathrm{F} 814 \mathrm{~W}$ to the corresponding Johnson-Cousins (Bessell 1990) magnitudes (in $V$ and $I$ ), following the prescriptions of Holtzman et al. (1995) with updates from Dolphin (2000b).

Since the post-explosion images were all obtained at the same epoch in each band and without any dithering between exposures, we straightforwardly input all of the individual exposures into HSTphot. The output from the package automatically includes the transformation from flight-system F555W and F814W to Johnson-Cousins $V$ and $I$.

Table 3 lists the photometry estimated for the SN and for the progenitor candidate.

\section{THE PROGENITOR OF SN 2008CN}

To further analyze the progenitor candidate, we must convert its observed brightness and color into intrinsic properties, such as luminosity and effective temperature. Doing so, we can attempt to place the star on a Hertzsprung-Russell (HR) diagram and draw some conclusions about its evolutionary state and initial mass.

From our adopted values for the distance and extinction to the $\mathrm{SN}$, we find that the absolute magnitudes of the progenitor candidate are $M_{V}^{0}=-7.31 \pm 0.21$ and $M_{I}^{0}=-8.29 \pm 0.23$. These values and the resulting intrinsic color, $(V-I)_{0}=0.98 \mathrm{mag}$, are more consistent with a supergiant star of spectral type $G$ than a much cooler RSG. If this star is the progenitor, it is far more yellow than the RSG progenitors so far identified, which would be an unusual, although not necessarily unprecedented, result. We note that $\mathrm{Li}$ et al. (2005) isolated a yellow supergiant as the progenitor of the high-luminosity SN II-P 2004et in NGC 6946 (but see Smartt et al. 2009). SN 2004et appears to have been somewhat unusual in its photometric and spectroscopic properties (Li et al. 2005; Sahu et al. 2006). Furthermore, the locus of the progenitor of SN 2006ov in M61 on the HR diagram may also indicate that the star was more yellow than red $(\mathrm{Li}$ et al. 2007; Prieto et al. 2008, although, again, see Smartt et al. 2009).

The metallicity at the SN site is likely solar. We determine this to be the case, based on the position of the $\mathrm{SN}$ in the host galaxy and the result derived from the typical central metallicity $(12+\log [\mathrm{O} / \mathrm{H}]=8.99 \pm 0.24)$ and gradient $\left(-0.06 \mathrm{dex} \mathrm{kpc}^{-1}\right)$ for a sample of 24 late-type spiral galaxies (Zaritsky et al. 1994). Thus, for the projected distance from the center of NGC 4603 to $\mathrm{SN} 2008 \mathrm{cn}(3.8 \mathrm{kpc})$, we estimate $12+\log [\mathrm{O} / \mathrm{H}]=8.76 \pm$ 0.24 , which is comparable to the solar value $(12+\log [\mathrm{O} / \mathrm{H}]=$ $8.66 \pm 0.05$; Asplund et al. 2005).

For solar metallicity, the derived color for the star corresponds to an effective temperature $T_{\text {eff }}=5200 \pm 300 \mathrm{~K}$ (the uncertainty

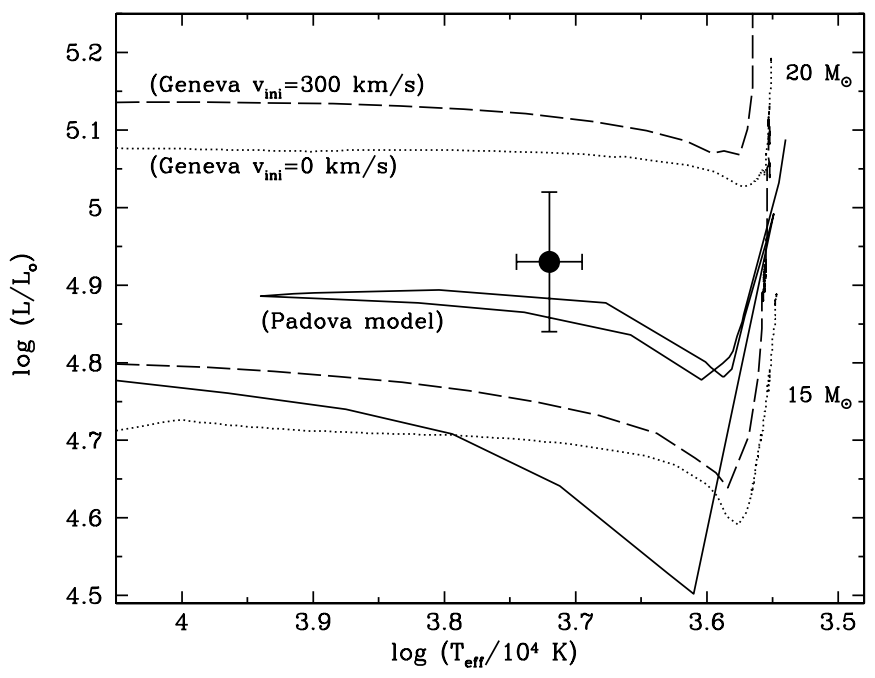

Figure 4. Hertzsprung-Russell diagram showing the bolometric luminosity, $L_{\mathrm{bol}}$, and effective temperature, $T_{\mathrm{eff}}$, for the candidate progenitor of SN $2008 \mathrm{cn}$ (filled circle). We have assumed a reddening $E(V-I)_{\text {tot }}=0.44$ mag and a distance modulus $\mu_{0}=32.61 \pm 0.20 \mathrm{mag}$ (Newman et al. 1999) for the star. Model stellar evolutionary tracks for a solar metallicity $(Z=0.02)$ are also shown for a range of masses and a rotation of $v_{\text {ini }}=0 \mathrm{~km} \mathrm{~s}^{-1}$ (i.e., no rotation; solid line for the $15 M_{\odot}$ track from Bressan et al. 1993 and dotted lines for the tracks from Hirschi et al. 2004) and $v_{\text {ini }}=300 \mathrm{~km} \mathrm{~s}^{-1}$ (dashed lines, Hirschi et al. 2004).

is a conservative estimate). For an assumed surface gravity $\log g=+0.5$, according to the Kurucz Atlas 9 models (Kurucz 1993), the bolometric correction to the absolute magnitude is -0.29 mag. Thus, the progenitor candidate has an absolute bolometric luminosity $L_{\text {bol }}=10^{(4.93 \pm 0.10)} L_{\odot}$ (assuming the Sun's absolute bolometric magnitude is 4.74).

The resulting locus for the star on a HR diagram is shown in Figure 4. For comparison we show evolutionary tracks for massive stellar models at solar metallicity. We first consider models without rotation and with rotation $v_{\text {ini }}=$ $300 \mathrm{~km} \mathrm{~s}^{-1}$, shown as dotted and dashed lines (respectively) in Figure 4 (Hirschi et al. 2004; hereafter referred to as the Geneva group tracks). Stellar rotation can have a profound effect on the inferred nature of the massive SN progenitors and on various observed characteristics of the explosion, particularly at higher masses, specifically, $15-25 M_{\odot}$. The Geneva group tracks have been computed in steps of 3-5 $M_{\odot}$. The locus for the progenitor candidate lies in between two sets of these models. A determination of the initial mass for the progenitor candidate therefore requires interpolation between these mass steps. Our "by eye" estimate is an initial mass of $18 \pm 2 M_{\odot}$. 
However, although the star appears to have a luminosity comparable to the RSG stage of the Geneva group tracks, the fact that it appears to be a yellow supergiant would require that it had undergone a "blue loop" away from the RSG branch. A blue loop could result from instabilities which occur in between the core He-burning and the shell H-burning stages, due to, for example, increasing $\mathrm{H}$ abundance, contraction of the $\mathrm{H}$ envelope as a consequence of an expansion of the He core, or a thermal non-equilibrium response of the stellar envelope to the variation of the core luminosity (Kippenhahn \& Weigert 1994; Xu \& Li 2004, and references therein). However, in the Geneva group tracks a late "blue" excursion is missing at masses between 12 and $20 M_{\odot}$, since these models still have most of their H-rich envelope at the termination of their calculation. The lack of a clear blue loop entails only a slight deviation from the Hayashi track, and this may sometimes occur only during the central C-burning phase (R. Hirschi and S. Ekström 2009 , private communication). For the Geneva group models with rotation, blue loops may occur for masses well above $15 M_{\odot}$ and below the limit for stars evolving to the Wolf-Rayet phase. For this reason, we also consider the Bressan et al. (1993) evolutionary tracks (hereafter referred to as the Padova group tracks). For these tracks, the blue loop is present at lower initial masses, such as $15 M_{\odot}$ (the solid line in Figure 4). However, we note that these models do not include rotation. Comparing the star's intrinsic properties with the Padova group track, the star's initial mass is consistent with $15 \pm 2 M_{\odot}$. Regardless of the evolutionary models, the biggest contributions to the uncertainty in this mass estimate come from the photometric uncertainties for such a relatively faint star, the uncertainties in the extinction, and the uncertainties in the bolometric correction and effective temperature for a yellow supergiant.

As can be seen, the inferred initial mass of the progenitor candidate for SN $2008 \mathrm{cn}$, based on the available evolutionary models, is within the range of progenitor initial masses found so far for SNe II-P (Smartt et al. 2009), albeit on the high side of that range. Considering that SN 2008cn appears to be a member of the high-luminosity SN subclass (see Section 2), this estimate of its progenitor's initial mass adds a valuable additional statistic, previously only provided, possibly, by SN 2004et (Li et al. 2005).

Of course, until now we have only been considering the progenitor candidate as a single star. The output from HSTphot, in addition to source position, flight-system magnitudes, and uncertainties, also contains the "sharpness" of the identified objects (see Dolphin 2000a for further details), which indicates the reliability that a detected source is indeed point like. As Leonard et al. (2008) recommend, a "good star" can be considered one where the value of this parameter is between -0.3 and +0.3 . From our runs of HSTphot on both F555W and F814W images, the overall sharpness of the progenitor candidate's profiles fell within these recommended limits, -0.036 in F555W and -0.184 in F814W. Additionally, the results also indicate that the "object type" flag is "1," further indicating that the source is likely stellar (see Dolphin 2000a). However, at the distance of the host galaxy, 33.3 Mpc (Newman et al. 1999), a single HST/WFPC2 pixel (at $0^{\prime} .05$ pixel $^{-1}$ ) is $\sim 8$ pc. Within this space a large number of stars could be contained, possibly even an unresolved stellar cluster. Although our progenitor candidate is quite luminous in $V$ and $I$, we can safely discount the progenitor candidate as a compact cluster, based on luminosity arguments (see Bastian et al. 2005; Crockett et al. 2008).



Figure 5. Hertzsprung-Russell diagram showing an example of a blue (B0 I spectral type, filled square) and a red (M1 I spectral type, filled circle) supergiant, which, when combined, result in the luminosity $L_{\mathrm{bol}}$ and $T_{\text {eff }}$ (dotted lines) estimated for the progenitor candidate of SN $2008 \mathrm{cn}$ (filled triangle). For comparison, evolutionary tracks with $Z=0.02$ and $v_{\text {ini }}=300 \mathrm{~km} \mathrm{~s}^{-1}$ (solid lines; Hirschi et al. 2004) are shown. The vertical dashed lines indicate the $T_{\text {eff }}$ for the input blue and red supergiants.

The yellow color of the SN progenitor could be the result of the blending of two or more stars into a single stellar profile. We attempted to deconvolve the yellow supergiant as a possible unresolved red and blue star combination, performing synthetic photometry using the package synphot within IRAF/STSDAS of model stellar spectra for blue supergiants (O8-B2 spectral types) and red supergiants (K3-M4 spectral types). The adopted values of effective temperature, $V-I$ color, and bolometric correction ascribed to these spectral types were obtained from Levesque et al. (2005), Humphreys \& McElroy (1984), and Kurucz (1993). In Figure 5, we show an example of one of these combinations, in comparison with the Geneva group tracks with rotation: the superposition of a $M_{\mathrm{ini}} \approx 40 M_{\odot}$ B0 I star and a $M_{\text {ini }} \approx 15 M_{\odot}$ M1 I star. From such a combination we can reproduce the intrinsic properties of the "yellow" star. Thus, a red supergiant with an initial mass of $15 M_{\odot}$ could be "hidden" within the superposition of the light from one or more brighter, bluer stars. Such a red star, in principle, could instead be the progenitor of SN $2008 \mathrm{cn}$.

Furthermore, Prieto et al. (2008) have found two eclipsing binaries containing massive yellow supergiants, one in the Small Magellanic Cloud and one in Holmberg IX. These authors point out that such an interacting binary could slow down the evolutionary transition from blue to red supergiant for the stars in the system, allowing the primary star to explode as a SN while still yellow. They offer these binaries as possible analogs for the progenitors of SNe 2004et and 2006ov. In a similar vein, it is possible that the yellow progenitor candidate for SN $2008 \mathrm{cn}$ is a similar close, massive binary system. Both the brightness and color of the binary in Holmberg IX (Prieto et al. 2008) are remarkably similar to those of the SN $2008 \mathrm{cn}$ progenitor candidate.

To that end, we are fortunate in the case of SN $2008 \mathrm{cn}$ that, for the first time in the study of the progenitor of a SN II-P, pre-explosion images exist at various epochs in two bands (Cohen et al. 1995 investigated the variability of the progenitor of the Type IIb 1993J in M81 and did not find any). We can therefore search for variability in the brightness of the 


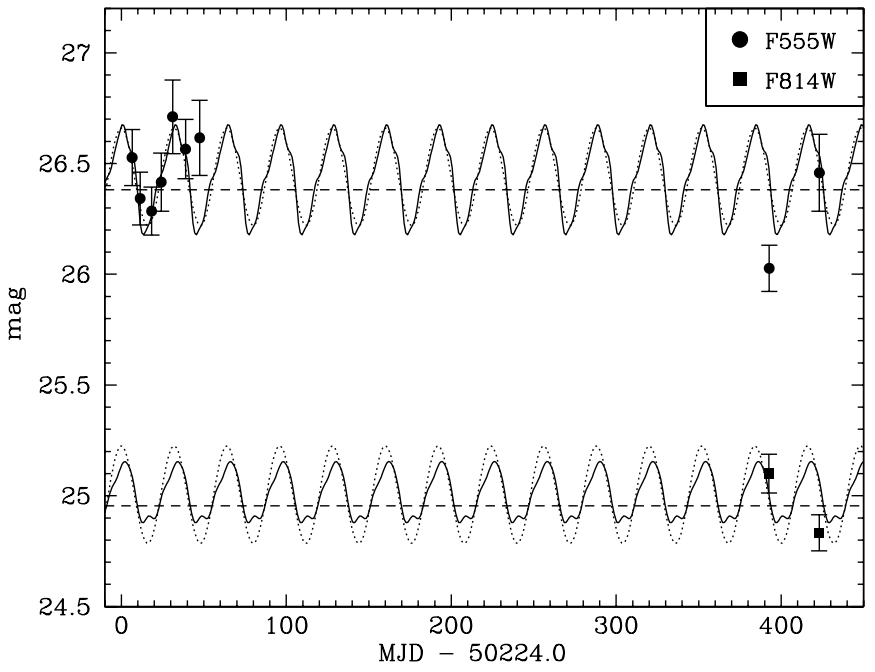

Figure 6. HST F555W ( $\sim V$; filled circles) and F814W ( $\sim I$; filled squares) magnitude vs. epoch for the progenitor candidate of SN $2008 \mathrm{cn}$. Each point is the average magnitude at each epoch in each band. Dashed lines indicate the average magnitude in each band. Model sinusoidal curves (simply representing an eclipsing binary system with components of equal brightness, solid lines) are also shown. The "fits" for the model curves were made purely by eye. See the text for discussion. Noticeable deviations from the model exist at some epochs.

progenitor candidate. In Figure 6, we show as a function of time the flight-system F555W and F814W magnitudes, which we obtained by running HSTphot on all CR-SPLIT pairs in each band at each epoch. As one can see, the progenitor candidate may marginally show variation in brightness in each band. Such variability would have occurred within 11-12 yr prior to explosion, when the pre-SN observations were made. In Figure 6 , we show for comparison model eclipses (simple sinusoids) in each band with period $\sim 64$ days and amplitude $0.22 \mathrm{mag}$ (solid curves), similar in shape to the eclipses for the binary in Holmberg IX (Prieto et al. 2008). By eye, this set of model light curves provides a reasonable representation of the apparent variability. We also note that, from a simple $\chi^{2}$ analysis, we actually find a smaller distribution in the residuals between data points and the average magnitude in each band for the progenitor candidate (dashed lines in Figure 6) than the simple varying model. This is not surprising, since the star is faint (average $V=26.41 \mathrm{mag}$ ) and the photometric uncertainties are large (the star is detected in each of the CR-SPLIT pairs at only $3-4 \sigma)$.

Finally, we also consider the ramifications if the candidate is $n o t$ the progenitor of SN $2008 \mathrm{cn}$. If the progenitor has not been detected in the pre-SN HST images, the upper limits in each band provide limits on the brightness, color, and initial mass of any undetected star. As stated above, no other point sources could be isolated in the pre-explosion $H S T$ images within a $3 \sigma$-radius $(\sim 5$ pixels) circle around the progenitor candidate position. We therefore input artificial stars with $27 \mathrm{mag} \lesssim V \lesssim 29 \mathrm{mag}$ (i.e., with brightnesses fainter than the progenitor candidate) and colors $1.5 \mathrm{mag} \lesssim V-I \lesssim 2.8 \mathrm{mag}$ for an assumed RSG at random positions within a radius of 10 pixels around the candidate's position using option 64 within HSTphot (see the resulting upper limits in Table 3). Applying the same total extinction as assumed for the yellow supergiant progenitor candidate and a corresponding bolometric correction of $-1.25 \mathrm{mag}$ appropriate for RSGs (Levesque et al. 2005), we find an upper limit to an undetected progenitor's bolometric luminosity of $L_{\text {bol }} \lesssim 10^{(4.65 \pm 0.23)} \mathrm{L}_{\odot}$. To convert this luminosity limit to an upper limit on initial mass, we considered the luminosities

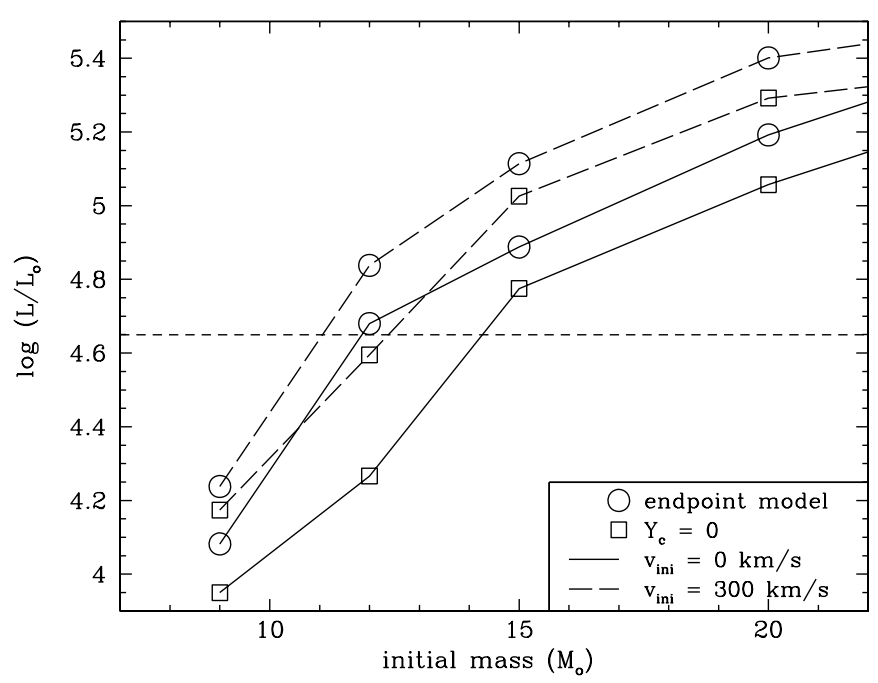

Figure 7. Initial mass vs. endpoint luminosity of the stellar models (Hirschi et al. 2004) for $Z=0.02$ at $v_{\text {ini }}=0 \mathrm{~km} \mathrm{~s}^{-1}$ (solid lines) and $v_{\text {ini }}=300$ $\mathrm{km} \mathrm{s}^{-1}$ (dashed lines). For each mass the luminosities corresponding to the endpoint of the model (open circles) and the end of the core He-burning phase (open squares) are shown. The short-dashed line indicates the upper luminosity limit for any red supergiant (in the range $27 \mathrm{mag} \lesssim V \lesssim 29 \mathrm{mag}$, $1.5 \mathrm{mag} \lesssim V-I \lesssim 2.8 \mathrm{mag}$ ) undetected near the site of SN $2008 \mathrm{cn}$.

of stellar models with rotation at solar metallicity, in the mass range 9-25 $M_{\odot}$ (Hirschi et al. 2004), at both the endpoints of the models and at the end of core He burning (see Smartt et al. 2009).

The results are shown in Figure 7. The upper limit to any undetected RSG progenitor's luminosity, in the extreme case in which such a star is at the end of the core He burning and without rotation at explosion, implies that it would have $M_{\text {ini }} \lesssim 14 M_{\odot}$. Considering, instead, what we believe is a physically more realistic scenario of explosion at the model endpoint luminosity for a rotating star, an undetected progenitor would have $M_{\text {ini }} \lesssim 11 M_{\odot}$. We note that both limits are also consistent with the results for other SNe II-P (Smartt et al. 2009). However, given the correlations between the main physical parameters for SNe II-P (see, e.g., Hamuy 2003; Pastorello 2003; Nadyozhin 2003), we might expect that high-luminosity $\mathrm{SNe}$ produce massive ejecta with high nickel masses $\left(M_{\mathrm{Ni}}\right)$ and, consequently, have high-mass progenitors. For example, Pastorello (2003), using a semianalytical model (Zampieri et al. 2003), estimated that the progenitor of SN $1996 \mathrm{~W}$ had an initial mass of $15.2_{-3.2}^{+6.4} M_{\odot}$. Since SN $2008 \mathrm{cn}$ also appears to be of the high-luminosity subclass, we might expect the $M_{\mathrm{Ni}}$ from this object, and therefore its progenitor mass, to also be relatively high (unfortunately, given the limited data available to us for SN $2008 \mathrm{cn}$, we cannot estimate the actual $M_{\mathrm{Ni}}$ here). Such expected high masses for the high-luminosity SN II-P progenitors stand at odds with the relatively low upper limits that we have derived for an undetected progenitor of SN $2008 \mathrm{cn}$.

That we cannot definitively identify the yellow supergiant candidate as the SN $2008 \mathrm{cn}$ progenitor most likely results from the fact that this is the most distant SN II-P (at 33.3 $\mathrm{Mpc}$ ) for which anyone has attempted the identification of a progenitor in pre-SN archival images. In fact, it is only due to the extraordinarily deep pre-explosion $H S T$ archival data that we are even able to attempt this. To explore the effects of the distance further, we estimated a limit in distance beyond which we can no longer confidently identify single stars in HST images at the typical spatial resolution of the WFC chip (it is relatively rare that a SN site will be located in archival PC chip 
images, due to that chip's much smaller area). We note that we expect the massive, RSG progenitors of SNe II-P to be found in relatively crowded environments with other massive starsthis, in fact, is generally borne out from detailed studies of these progenitors (see Smartt et al. 2009, and references therein). We conducted a simple test using the $H S T$ images containing the progenitor of the comparatively nearby and well-studied SN II-P 2003gd (Van Dyk et al. 2003). SN 2003gd, in the host galaxy M74 at $7.2 \mathrm{Mpc}$, was most likely the explosion of a RSG with initial mass $\sim 8 M_{\odot}$, identified by Van Dyk et al. (2003) as "Star A" in an archival WFPC2 WF2 chip image in F606W (and later confirmed by Smartt et al. 2004). Nearby $(\sim 10.5 \mathrm{pc})$ to Star A is a bluer object, "Star B." We artificially degraded the spatial resolution of the SN 2003gd pre-explosion image until it was no longer possible to distinguish between the two individual stars in the image. The resulting degraded resolution corresponds to the equivalent resolution of an image of a galaxy at a distance of $\sim 20 \mathrm{Mpc}$. We conclude, therefore, that for galaxies which host SNe II-P at distances $\gtrsim 20 \mathrm{Mpc}$, such as NGC 4603, it becomes far more difficult to identify unambiguously their (RSG) progenitors. This distance sets a practical limit on using even the high spatial resolution of HST images to address the problem of progenitor identification.

\section{CONCLUSIONS}

We have shown, based on a limited data set, that the SN II-P 2008cn in NGC 4603 appears to be of the highluminosity subclass, similar to SN 1996 W (Pastorello 2003), SN 2003hn (Krisciunas et al. 2009), and possibly SN 2004et (Sahu et al. 2006). Its $V$-band luminosity some weeks after explosion is $\sim 0.5$ mag brighter than that of, for example, the normal SN II-P 1999em (Hamuy et al. 2001; Leonard et al. 2002; Elmhamdi et al. 2003). The progenitors of normal SNe II-P have all been determined or inferred to be RSGs (Smartt et al. 2009). It is unclear at this point, due to a relative lack of examples, whether high-luminosity SNe II-P, such as SN $2008 \mathrm{cn}$, also arise from RSGs.

High-precision relative astrometry has been employed to identify a candidate progenitor for SN $2008 \mathrm{cn}$, based on a comparison of high spatial resolution HST imaging both of the SN at late times and of the host galaxy containing the $\mathrm{SN}$ site prior to explosion. Comparison of the difference between the SN and candidate positions with the astrometric uncertainties suggests that we may well have identified the SN progenitor, although this is not entirely certain. In estimating an initial mass for the progenitor candidate, we have been careful to transform the observed photometry into intrinsic stellar quantities, specifically the bolometric luminosity and the effective temperature of the star. We have also considered for comparison the most recent available stellar evolutionary models for massive stars, including those with rotation (in particular, the Geneva group models; the Padova group models do not include rotation). From this we have estimated an initial mass for the progenitor of SN $2008 \mathrm{cn}$ of $15 \pm 2 M_{\odot}$, which is within the initial mass range for SN II-P progenitors, based on all previous attempts that have been made to identify the star (Smartt et al. 2009).

However, the star's color is more yellow than we would expect. This unusual color could arise from the star exploding while experiencing an evolutionary blue loop, which is theoretically not expected to occur. Alternatively, the stellar profile could consist of two or more stars in a blend, with a possible combination of one or more blue and red supergiants resulting in the yellow color. In this case, a blended stellar profile could "conceal," for example, a $\sim 15 M_{\odot}$ RSG, which could nominally be the progenitor. Additionally, the blend could be a massive, interacting binary, analogous to the yellow supergiants recently found in extragalactic eclipsing binary systems (Prieto et al. 2008). That SN 2008cn, like SN 2004et, is a high-luminosity SN II-P makes this a particularly intriguing possibility. The progenitor of SN 2004et has been identified as a yellow supergiant $(\mathrm{Li}$ et al. 2005), also coincidentally with $M_{\text {ini }} \approx 15 M_{\odot}$, although this identification is controversial (see Smartt et al. 2009). Close interaction in such a massive binary system could prevent the primary from evolving to the RSG phase, allowing the star to explode as a yellow supergiant. SN $2008 \mathrm{cn}$ could well represent an additional example of what is likely a relatively rare event.

Establishing the precise position of the faint progenitor candidate in the pre-explosion archival images has proven to be challenging, especially with the presence of the two brighter stars near the fainter candidate and the relatively large distance to the host galaxy. The slight excess in the positional difference in declination, relative to the positional errors, prevents us from definitively assigning this star as the progenitor. If the candidate yellow supergiant is not the SN 2008cn progenitor, then the upper limits to detecting any star within $\sim 10$ pixels $(\sim 80$ pc) of this candidate result in a conservative upper limit to the initial mass of a RSG progenitor of $M_{\text {ini }} \lesssim 14 M_{\odot}$. Ultimately, the best test of the nature of the SN $2008 \mathrm{cn}$ progenitor is to observe this field again with HST (or the James Webb Space Telescope) several years later, when the SN has faded to nondetectability. The definitive indication would be if the yellow star has vanished, or is significantly diminished in brightness, or has survived intact.

Financial support for this work was provided by NASA through grants AR-10952, AR-11248, and GO-11119 from the Space Telescope Science Institute, which is operated by Associated Universities for Research in Astronomy, Inc., under NASA contract NAS 5-26555. We acknowledge the National Science Foundation (NSF) through grant AST-0306969 for support of the Carnegie Supernova Program. N.E.R. thanks Andrew Dolphin for his help with HSTphot, Philip Massey and Raphael Hirschi for their help with the supergiant parameters and models, Artemio Herrero for stimulating discussions, Stefano Benetti for his help in the determination of SN parameters, and Andrea Pastorello and the Padova-Asiago SN Group for access to the data for SN 1996W. M.H. acknowledges support from FONDECYT through grant 1060808, the Millennium Center for Supernova Science through grant P06-045-F funded by "Programa Bicentenario de Ciencia y Tecnología de CONICYT" and "Programa Iniciativa Científica Milenio de MIDEPLAN," Centro de Astrofísica FONDAP 1501000, and Center of Excellence in Astrophysics and Associated Technologies (PFB 06). This publication makes use of data products from the Two Micron All Sky Survey, which is a joint project of the University of Massachusetts and the Infrared Processing and Analysis Center/California Institute of Technology, funded by NASA and the NSF.

Facilities: HST (WFPC2), Magellan: Baade (IMACS), Swope

\section{REFERENCES}

Asplund, M., Grevesse, N., \& Sauval, A. J. 2005, in ASP Conf. Ser. 336, Cosmic Abundances as Records of Stellar Evolution and Nucleosynthesis, ed. T. G. Barnes, III \& F. N. Bash (San Francisco, CA: ASP), 25 Barbon, R., Ciatti, F., \& Rosino, L. 1979, A\&A, 72, 287 
Bastian, N., Gieles, M., Efremov, Y. N., \& Lamers, H. J. G. L. M. 2005, A\&A, 443, 79

Bessell, M. S. 1990, PASP, 102, 1181

Bressan, A., et al. 1993, A\&AS, 100, 647

Cardelli, J. A., Clayton, G. C., \& Mathis, J. S. 1989, ApJ, 345, 245

Clocchiatti, A., et al. 1996, AJ, 111, 1286

Cohen, J. G., Darling, J., \& Porter, A. 1995, AJ, 110, 308

Crockett, R. M., et al. 2008, ApJ, 672, L99

Dolphin, A. E. 2000a, PASP, 112, 1383

Dolphin, A. E. 2000b, PASP, 112, 1397

Elmhamdi, A., et al. 2003, MNRAS, 338, 939

Fruchter, A. S., \& Hook, R. N. 2002, PASP, 114, 144

Gal-Yam, A., \& Leonard, D. C. 2009, Nature, 458, 865

Gal-Yam, A., et al. 2007, ApJ, 656, 372

Hamuy, M. 2003, ApJ, 582, 905

Hamuy, M., et al. 2001, ApJ, 558, 615

Hirschi, R., Meynet, G., \& Maeder, A. 2004, A\&A, 425, 649

Holtzman, J. A., et al. 1995, PASP, 107, 1065

Humphreys, R. M., \& McElroy, D. B. 1984, ApJ, 284, 565

Kippenhahn, R., \& Weigert, A. 1994, Stellar Structure and Evolution (Berlin: Springer)

Krisciunas, K., et al. 2009, AJ, 137, 34

Kurucz, R. L. 1993, CD-ROM No. 13, Smithsonian Astrophysical Observatory, 18

Leonard, D. C., et al. 2002, PASP, 114, 35

Leonard, D. C., et al. 2008, PASP, 120, 1259

Levesque, E. M., et al. 2005, ApJ, 628, 973
Li, W., Van Dyk, S. D., Filippenko, A. V., \& Cuillandre, J.-C. 2005, PASP, 117, 121

Li, W., et al. 2006, ApJ, 641, 1060

Li, W., et al. 2007, ApJ, 661, 1013

Li, W., et al. 2008, Central Bureau Electronic Telegrams, 1397, 1

Marek, A., \& Janka, H.-Th. 2009, ApJ, 694, 664

Martin, R., \& Monard, L. A. G. 2008, Central Bureau Electronic Telegrams, 1385,1

Nadyozhin, D. K. 2003, MNRAS, 346, 97

Newman, J. A., et al. 1999, ApJ, 523, 506

Pastorello, A. 2003, PhD thesis, Univ. Padua

Prieto, J. L., et al. 2008, ApJ, 673, L59

Sahu, D. K., Anupama, G. C., Srividya, S., \& Muneer, S. 2006, MNRAS, 372, 1315

Schlegel, D. J., Finkbeiner, D. P., \& Davis, M. 1998, ApJ, 500, 525

Smartt, S. J. 2002, Ap\&SS, 281, 187

Smartt, S. J., et al. 2004, Science, 303, 499

Smartt, S. J., et al. 2009, MNRAS, 395, 1409

Stetson, P. B. 1987, PASP, 99, 191

Stritzinger, M., \& Morrell, N. 2008, Central Bureau Electronic Telegrams, 1393, 1

Van Dyk, S. D., et al. 1999, AJ, 118, 2331

Van Dyk, S. D., et al. 2000, PASP, 112, 1532

Van Dyk, S. D., et al. 2003, PASP, 115, 1289

Xu, H. Y., \& Li, Y. 2004, A\&A, 418, 213

Zampieri, L., et al. 2003, MNRAS, 338, 711

Zaritsky, D., Kennicutt, R. C., Jr., \& Huchra, J. P. 1994, ApJ, 420, 87 